\title{
Ist die Zukunft der Medizin weiblich?
}

\author{
B. von Castelberg*
}

\footnotetext{
* Mitglied der Experten/-innengruppe «Zukunft Medizin Schweiz»
}

Korrespondenz: Dr. med. Brida von Castelberg Maternité Inselhof Triemli Birmensdorferstrasse 501 CH-8063 Zürich
90\% aller Pflegenden, 100\% aller Hebammen und Kinderkrankenschwestern und 80\% aller Physiotherapeutinnen sind und waren schon immer weiblich. Wenn man die Medizin also definiert als den Prozess von Krankheitsprävention, von Diagnose, Therapie, Rehabilitation und Pflege, so sind die meisten der in diesem System agierenden Personen weiblich. Warum also die Frage nach einer weiblichen Zukunft, oder anders gefragt: Warum wird die heutige Medizin als männlich empfunden?

Die zweite Feststellung ist historischer Natur: Frauenberufe waren bis am Anfang des 20. Jahrhunderts auf die Zeit zwischen Erwachsenwerden und Familiengründung beschränkt, und da keine Karriere geplant war, waren die Frauenberufe nicht oder schlecht bezahlt und nicht prestigeträchtig. Es waren Berufe, die später in der Familie genutzt werden konnten, wie Pflege, Kindererziehung, Altenbetreuung oder Service. Daneben wurde von Frauen dieser Zeit ganz selbstverständlich unbezahlte Freiwilligenarbeit geleistet. Nach der Eheschliessung prägte dann die uneigennützige Verantwortung für die Kindererziehung die weibliche Ethik.

Die Rollenzuteilung in der Medizin lässt sich nach wie vor (und etwas vereinfachend) von diesen historisch gegebenen Tatsachen ableiten: Dabei steht «curing» für das männliche, «caring» für das weibliche Prinzip in der Medizin.

Curing ist, was man gemeinhin vom Arzt erwartet: eine Krankheit aufgrund der Symptome diagnostizieren, notwendige Untersuchungen veranlassen und in nützlicher Frist durch eine richtige Therapie die Krankheitssymptome zum Verschwinden bringen. Heilung bzw. Symptomfreiheit ist das Ziel; je rascher der Erfolg, desto erfolgreicher und ruhmvoller der Arzt.

Davon unterscheidet sich klar der CaringAspekt: Caring ist nicht eine reine Tätigkeit, sondern vor allem eine Haltung gegenüber einem Menschen, eine Haltung von Wertschätzung und Empathie. Es steht nicht eine Krankheit oder ein Symptom, sondern ein kranker Mensch im Zentrum des Therapieansatzes, und nicht nur eine Krankheit muss erkannt, sondern auch Leiden müssen ernst genommen und behandelt werden.

\section{Zukunft Medizin Schweiz}

Die Schweizerische Akademie der Medizinischen Wissenschaften (SAMW) engagiert sich seit mehreren Jahren im Projekt «Zukunft Medizin Schweiz» (früher «Neu-Orientierung der Medizin»). Seit Anfang 2002 wird das Projekt von der FMH und den Medizinischen Fakultäten der Schweiz mitgetragen. In einer ersten Phase soll eine Experten/-innengruppe unter Leitung von Prof. Dieter Bürgin aus Basel die Ziele und Grenzen der Medizin für die Schweiz formulieren. Die Steuerungsgruppe mit Vertretern der Auftraggeber wird am 27. August 2003 im Rahmen eines «Forums» die wesentlichen inhaltlichen Ergebnisse dieser ersten Phase vorstellen und zur Diskussion stellen.

In den nächsten Monaten werden sich Mitglieder der Experten/-innengruppe in loser Folge in dieser «Kolumne» zu Wort melden und von der Arbeit bzw. den Diskussionen in der Gruppe sowie von ihren eigenen Erfahrungen und Überlegungen berichten.

\section{La médecine en Suisse demain}

Depuis quelques années, l'Académie Suisse des Sciences Médicales (ASSM) s'engage pour le projet «L'avenir de la médecine en Suisse» (initialement appelé «Nouvelle orientation de la médecine»). Depuis l'année dernière, le projet est également soutenu par la FMH et les facultés de médecine suisses. Dans une première phase, un groupe d'expert(e)s sous la direction du Prof. Dieter Bürgin de Bâle définira les buts et les limites de la médecine en Suisse. Le groupe de pilotage constitué de représentants des mandants présentera le 27 août 2003, dans le cadre d'un «forum», les principaux résultats de cette première phase et ouvrira le débat.

Dans les prochains mois, les membres du groupe d'expert(e)s s'exprimeront de temps à autre dans cette rubrique et feront part de leur travail, de leurs discussions, de leurs expériences ainsi que de leurs réflexions. 
Bei der Forderung nach Empowerment der Patienten durch Aufklärung und zunehmende Mitbestimmung der Patienten in der Therapie wird vermehrt auch eine Caring-Medizin gefragt sein, wo Arzt und Patient gemeinsam verschiedene Therapien und Alternativen individuell auf eine Person und ihr Umfeld bezogen abwägen und wählen können.

Betrachten wir die unzähligen medizinischen Sendungen am Fernsehen, seien es informativ fachliche oder auch Soapserien (und sie machen viele Stunden pro Woche aus), so sind vor allem schnelle spektakuläre Therapien und meist männliche Protagonisten gefragt. Lebensrettende Massnahmen nach Unfall, Herzoperationen, Transplantationen, Roboteroperationen usw. prägen in den Medien das Bild der Medizin. Es sind dies klassische Curing-Situationen.

Wenn sich Frauen in der Berufswahl klar gegen eine Familie und Kinder entscheiden, so stehen ihnen fast die gleichen Karrierechancen offen wie ihren männlichen Kollegen. Wenn sich eine Frau jedoch die Option Familie offenhalten will, werden bereits gewisse medizinische Spezialitäten in der Berufswahl ausgeschlossen und andere vorgezogen, welche nach einer Kinderpause einen erleichterten Wiedereinstieg möglich machen oder Arbeit in Teilzeitpensum erlauben. Es sind dies vor allem Fächer der Grundversorgung wie Allgemeinmedizin oder nichtoperative Fächer. Die spektakulären, operativen Fächer fallen dann oft von vornherein weg. Die gewählten Fächer sind jene, die weniger öffentliches Ansehen geniessen (Feld-, Waldund Wiesenarzt) und schlechte Einkommensmöglichkeiten bieten. Die von der Öffentlichkeit wahrgenommene Medizin ist also auch weiterhin männlich.

Obwohl gleich viele Frauen wie Männer das Medizinstudium beenden, werden nach wie vor 90\% der hohen Positionen in der Universität durch Männer besetzt und über 90\% der Medizinischen Chefärzte sind männlich, d.h., da wo über Forschungsinhalte entschieden oder medizinisches Wissen vermittelt wird, sind die Entscheidungsträger männlich. Dringend erforderlich ist eine gezielte Nachwuchsförderung für Frauen (inkl. Anpassen der Krippenöffnungszeiten an Arbeitszeiten) und ein Überdenken des derzeitigen Habilitationsverfahrens.

Ziel des Projektes «Zukunft Medizin Schweiz» ist es, neue Prioritäten in der Medizin zu setzen. Von entscheidender Bedeutung wird sein, ob die (männlichen) Spitzenpositionen in den Fakultäten und den öffentlichen Spitälern diese neuen Ziele akzeptieren und auch vermitteln werden. Dies wird nicht so sehr zu einer Feminisierung der Medizin, sondern zu einer wohltuenden Humanisierung der Medizin führen.

Bisher unter «Zukunft Medizin Schweiz» erschienen:

Bürgin D. Wohin steuert die Medizin? Schweiz Ärztezeitung 2003;84(13):578-9. 